\title{
Modeling echelle spectrographs
}

\author{
P. Ballester ${ }^{1}$ and M.R. Rosa ${ }^{2, \star}$ \\ ${ }^{1}$ European Southern Observatory, Karl-Schwarzschild-Str. 2, D-85748 Garching, Germany \\ e-mail: pballest@eso.org \\ ${ }^{2}$ Space Telescope - European Coordinating Facility Karl-Schwarzschild-Str. 2, D-85748 Garching, Germany \\ e-mail: mrosa@eso.org
}

Received February 6; accepted March 28, 1997

\begin{abstract}
A generic description of spectrographs based on first optical principles is developed. It incorporates offplane grating equations and rotations in three dimensions in order to adequately account for line tilt and order curvature. This formalism is validated by confronting the models for two actual spectrographs (UVES and CASPEC) with ray tracing results and arc lamp exposures. The versatility of these models for the control of instrument configurations, for the generation of calibration databases, and for the preparation of observations is shown. As an important application, we derive from this formulation various forms of the echelle relation which can be used to implement automatic wavelength calibration procedures. Finally, we discuss possible applications of such analytical models of astronomical instruments for calibration, data analysis and observatory operations.
\end{abstract}

Key words: instrumentation: spectrographs - methods: data analysis — techniques: image processing; spectroscopic

\section{Introduction}

The rapid evolution of detector and instrument technology has provided astronomers with the capabilities to acquire large amounts of high signal-to-noise, multi-dimensional observational data. In order to exploit optimally these data, the entire chain of the observation process from instrument configuration control through calibration, analysis and archival has to be tailored towards very high standards. In contemporary data calibration and analysis very little has been done so far to relate the optical layout and its engineering parameters with the performance on scientific targets and calibration sources. Even less use has been

\footnotetext{
Send offprint requests to: P. Ballester

* Affiliated to the Astrophysics Division of the Space Science Department of the European Space Agency.
}

made of the physical principles underlying the characteristics of a given instrument in predicting the performance to a large degree of accuracy (cf. Rosa 1995).

A number of developments make it more practical today to efficiently use optical models in the postobservational process (see Ballester 1996). First of all instrument configuration control, which is self evident for space missions, is now being enforced more strictly at ground based instruments. Second the development of service observing modes at major facilities like the Very Large Telescope (VLT) of the European Southern Observatory (ESO) necessitates the development of data quality control procedures. And third, the need to process large amounts of data in a homogeneous manner calls for robust automatic calibration techniques (Ballester 1994). First principle based analysis techniques have been applied for example in the algorithm to correct for the grating scatter in the HST Faint Object Spectrograph (FOS) (Rosa 1994), which was implemented in major data analysis environments (Bushouse et al. 1995). A model based solution to first order spectrograph dispersion relations has been used by Dahlem \& Rosa (1997) to assess the uncertainties inherent in low order polynomial fits for wavelength calibrations for the FOS. Another interesting case is the HST PSF model (e.g. Hasan \& Burrows 1994), which shows the relation between using a model of the optics to retrieve parameters for the refurbishing mission and its use to generate PSFs for deconvolution.

One of the most demanding cases of data calibration and analysis are 2D echelle spectra. Traditionally, they require complex data reduction procedures to cope simultaneously with both, the geometrical distortion of the raw data introduced by order curvature and line tilt, and with the spread of the signal across the tilted lines and between successive orders respectively (cf. Hensberge \& Verschueren 1990). Un-supervised wavelength calibration for these instruments can only be achieved by reducing to a minimum the information needed to start the calibration process. This requires the most efficient use of the a priori knowledge from the optical properties of the 
instrument under consideration. For instance the echelle relation is commonly employed to start the calibration process, and can in the simplest cases be determined with only 2 interactive line identifications. However, the validity of the simple echelle relation is limited to those cases where the detector rotation and optical aberrations are negligible, as pointed out for instance by Hall et al. (1994). Another approach is to use encoder values of the grating angles fed into analytic formulae for the dispersion relation, presented by Goodrich \& Veilleux (1988) for an in-plane spectrograph. However, a more general solution capable of handling off-plane situations has not been developed so far.

We study in this article the principles governing the determination of an accurate form of the echelle relation for off-plane echelle spectrographs, and analyze the applications of such models for the calibration of echelle spectrographs. In a first step we derive the characteristic equations of echelle spectrographs from first principles (Sect. 2 and Appendix A). This formalism is then confronted with the results of ray tracing analysis for the demanding case of an off-plane echelle spectrograph (Sect. 3). In Sect. 4 the application of models to the task of instrument configuration control is discussed and verified on actual data from an instrument that has been in service for a decade. In Sect. 5 and Appendix B methods derived from such optical models are introduced that allow one to reduce to a minimum the information needed to start the wavelength calibration process. Section 6 will be used to discuss possible applications of such analytical models for the calibration of astronomical instruments, advanced data analysis, and observation planning.

\section{Optical principles for spectrographs}

\subsection{Optical elements and geometry}

The elements of a model for echelle spectrographs are mirrors, lenses, gratings, prisms, grisms. Here we will focus solely on the geometric aspects, i.e. the $2 \mathrm{D}$-dispersion relation. Luminosity aspects brought about by the interference terms, e.g. the echelle blaze function, line spread functions, as well as geometrical vignetting or reflectivity and transmissivity of materials will be the subject of subsequent communications. The notations of Schroeder (1987) are followed for the definitions of angles and orientations (right-hand Cartesian frame).

A ray incident on an optical surface co-planar with the $x y$-plane has direction $(\alpha, \gamma)$ (see Fig. 1$)$ and projects onto the axes as

$$
\begin{aligned}
& x=\sin \alpha \cos \gamma \\
& y=\sin \gamma \\
& z=\cos \alpha \cos \gamma
\end{aligned}
$$

Conversely the angles $(\alpha, \gamma)$ are given by

$$
\begin{aligned}
& \alpha=\arctan \frac{x}{z} \\
& \gamma=\arctan \left(\frac{y}{\sqrt{x^{2}+z^{2}}}\right) .
\end{aligned}
$$

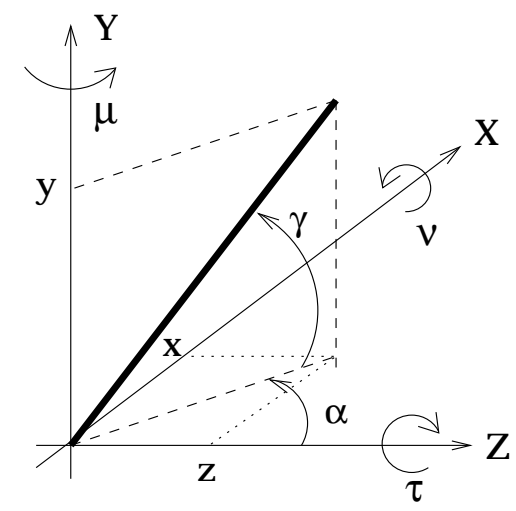

Fig. 1. Axes and angles notations

Since these angles are for incidences, their values are comprised in the interval $-\frac{\pi}{2}$ to $\frac{\pi}{2}$. The orientation of the optical surface is described by the rotation angle $\tau$ about the $z$ axis. The angles $\mu$ and $\nu$ denote rotations relative to $x$ and $y$ respectively. Referential changes are performed by applying the rotations in the following order:

$R=R_{-\tau / z} R_{-\nu / x} R_{-\mu / y}$

and the transpose matrix provides the opposite change of the referential:

$R^{T}=R_{\mu / y} R_{\nu / x} R_{\tau / z}$

where each rotation matrix is of the form:

$R_{\tau / z}=\left(\begin{array}{ccc}\cos \tau & \sin \tau & 0 \\ -\sin \tau & \cos \tau & 0 \\ 0 & 0 & 1\end{array}\right)$

\subsection{Diffraction by the echelle grating}

The direction of incident rays on an echelle grating is defined by the two angles $\left(\alpha_{\mathrm{E}}, \gamma_{\mathrm{E}}\right)$ (Schroeder 1987). The general grating equation can be written as

$\frac{m \lambda}{\sigma}=n^{\prime} \cos \gamma_{\mathrm{E}}^{\prime} \sin \beta_{\mathrm{E}}-n \cos \gamma_{\mathrm{E}} \sin \alpha_{\mathrm{E}}$

and

$n \sin \gamma_{\mathrm{E}}=n^{\prime} \sin \gamma_{\mathrm{E}}^{\prime}$

where $m$ is the order number, $\lambda$ the wavelength, $\sigma$ the groove separation in unit of length, $n$ and $n^{\prime}$ the refractive 
indices before and after the grating, $\alpha_{\mathrm{E}}$ and $\gamma_{\mathrm{E}}$ the incidence angles, and $\beta_{\mathrm{E}}$ and $\gamma_{\mathrm{E}}^{\prime}$ the direction of the diffracted rays.

For a reflection grating $n^{\prime}=-n$ and therefore $\gamma_{\mathrm{E}}^{\prime}=$ $-\gamma_{\mathrm{E}}$, hence

$\frac{m \lambda}{\sigma}=-n \cos \gamma_{\mathrm{E}}\left(\sin \beta_{\mathrm{E}}+\sin \alpha_{\mathrm{E}}\right)$.

One can note that using the projection equations it is possible to write the two grating Eqs. (9) and (10) as:

$n^{\prime} x^{\prime}=\frac{m \lambda}{\sigma}+n x$

$n^{\prime} y^{\prime}=n y$.

This formulation allows to introduce the grating in the model in the form of a matrix. However, since no simple optical equation allows to derive $z$ this term will need to be derived from the normalization relation:

$z=\sqrt{1-x^{2}-y^{2}}$.

\subsection{Echelle relation}

The echelle relation of a spectrograph derives from the echelle grating equation. For a constant $\alpha_{\mathrm{E}}$ and all rays corresponding to a constant product $m \lambda$ are diffracted in the direction $\beta_{\mathrm{E}}$. In a simplistic model one could expect that the lines of constant value $m \lambda$ project as straight lines in the detector planes and for a perfect alignment of the detector as, say, columns on the detector. This assumption is however limited by aberrations and rotation of the detector.

\subsection{Diffraction by the cross-disperser}

The cross-disperser is either a grating, a prism or a grism of low spectral resolution. It displaces successive orders of the echelle grating vertically with respect to each other. It is normally rotated by an angle $\tau_{\mathrm{CD}}=\pi / 2$ so that the dispersion equations of the cross-disperser needs to be applied after rotation to the referential of the cross-disperser. $\sigma_{\mathrm{CD}}$ denotes the groove separation for this grating.

\subsection{Planes, mirrors and grisms}

The refraction at a plane is given by Snell-Descartes law $n \sin \theta=n^{\prime} \sin \theta^{\prime}$.

The projection of an incident beam is given by Eq. (1) and the angle $\theta$ with the $z$ axis is:

$\cos \theta=\cos \gamma \cos \alpha$.

One can also express this relation using the direction cosine vector:

$x^{\prime}=n x / n^{\prime}$

$y^{\prime}=n y / n^{\prime}$

$z^{\prime}=\sqrt{1-x^{\prime 2}-y^{\prime 2}}$.
For $n^{\prime}=-n$ these are the equations for a mirror. This paper does not discuss grism based spectrographs although the above equations have been used to model such spectrographs. We indicate it here for the sake of completeness of the analytical framework. Grisms are represented by the association of a plane and a grating.

\subsection{Lenses and curved mirrors with positive power}

In the referential of the optical element the projections $(x, y)$ on the focal plane at focal distance $F$ are given by

$$
\begin{aligned}
& x_{\mathrm{d}}=F \tan \alpha \\
& y_{\mathrm{d}}=F \tan \gamma .
\end{aligned}
$$

Equations (4) and (5) allow to derive directly the values of $\tan \alpha$ and $\tan \gamma$ from the coordinates of the unit vector $(x, y, z)$.

\subsection{Projection onto detectors}

Translation and rotation of the detector array are applied to the vector point $(x, y)$ by

$x_{\mathrm{r}}=x_{\mathrm{d} 0}+x_{\mathrm{d}} \cos \left(\tau_{\mathrm{DET}}\right)+y_{\mathrm{d}} \sin \left(\tau_{\mathrm{DET}}\right)$
$y_{\mathrm{r}}=y_{\mathrm{d} 0}-x_{\mathrm{d}} \sin \left(\tau_{\mathrm{DET}}\right)+y_{\mathrm{d}} \cos \left(\tau_{\mathrm{DET}}\right)$

This rotation can be applied as a rotation matrix before entering the camera lens. This step is performed after the lens projection in order to determine the analytical form of the dispersion relation in the absence of detector rotation.

\subsection{Aberrations and chromatism}

In a complete optical train the above set of equations strictly apply only for on-axis rays and do not take into account field distortions, camera aberrations and wavelength dependencies of e.g. the focal lengths. In instruments like UVES (discussed below) these effects can account for discrepancies of several pixels at the detector.

Distortions are specific to the optical elements and layout, and are usually predictable and stable in time. As will be shown in Sect. 3 a model for a given instrument will typically match $99.5 \%$ using the physical description as developed above. For most applications it will not pay off to develop further the description by introducing off-axis optical equations. Instead the residuals can be corrected for by inserting at the proper location low order polynomial functions whose coefficients can for example be produced with the help of a ray tracing program.

In the following we will closely analyze the application of both, the exact (on axis) equations and a simplified analytical form respectively. Two notions of accuracy will be used. On the one hand the absolute accuracy of the model is limited by the optical effects not taken into account like e.g. aberrations and distortions. On the other hand the 
accuracy of the simplified analytical model will be limited by the numerical approximations performed to keep the analytical form simple (e.g. Taylor series expansions).

\section{Analytical model versus ray tracing}

In this section we use the general framework derived above to model the UVES spectrograph and compare the results with those from a Code $\mathrm{V}^{1}$ ray tracing analysis.

\subsection{UVES as a case study}

The UV Visual Echelle Spectrograph UVES (D'Odorico 1997), to see first light in 1999 on the VLT, is a two-arm cross-dispersed echelle spectrograph covering the wavelength range $300-500 \mathrm{~nm}$ (blue) and $420-1100 \mathrm{~nm}$ (red), with the possibility to use dichroics. The nominal resolution is 40000 for a $1^{\prime \prime}$ slit, the maximum resolution that can be attained with a narrow slit or image slicer is 120000 in the red and 90000 in the blue with 2-pixel sampling. The dioptric cameras offer fields with a diameter of $43.5 \mathrm{~mm}$ (blue) and $87 \mathrm{~mm}$ (red), to be recorded by baseline CCD detectors of $2048 \times 2048$ pixels of size $15 \mu \mathrm{m}$ in the blue arm and 2 or 4 such devices in the red arm. The instrument components are placed inside a passive enclosure which provides thermal isolation from the environment. The control and CCD electronics are located in temperature controlled cabinets outside the enclosure. All functions (filters, ADC etc.) are permanently on-board and remotely selectable without manual intervention. A continuous flow of liquid N2 coolant for the CCD's is supplied by an external vessel with an autonomy of at least 14 days. These measures are expected to lead to high stability and repeatability of calibrations both over short and extended periods of time.

UVES was selected as a case study because the offplane design is introducing a pronounced slit curvature, and therefore offers a demanding case for any modeling approach. Elaborate ray tracing results are available from the optical design phase. Moreover, since the design of this instrument is tailored towards ensuring a high degree of stability (see above), it makes it a perfect candidate for model based calibration and data analysis approaches.

\subsection{Modeling UVES}

For our study we use the optical design parameters of the red arm configuration of UVES with the 316 grooves $/ \mathrm{mm}$ cross-disperser. This configuration is described by

- an echelle reflection grating (31.6 grooves $/ \mathrm{mm}$ ). The beam enters at $\alpha_{\mathrm{E}}=-75$ degrees and $\gamma_{\mathrm{E}}=-0.8$ degrees. The output axis is oriented at $\beta_{\mathrm{E}}=-75$ degrees, $\gamma_{\mathrm{E}}^{\prime}=0.8$ degrees.

1 Code $\mathrm{V}$ is a trademark of Optical Research Associates, Pasadena, California.
- an a-focal system composed of two collimators of focal length $F_{1}=F_{2}=2.0$ meters. The system introduces field curvature in the direction of the dispersion of the echelle grating. In our model, field curvature is approximated by a polynomial.

- a reflecting cross-disperser (316 grooves $/ \mathrm{mm}$ ). The difference between incident and diffracted directions is $\beta_{\mathrm{CD}}-\alpha_{\mathrm{CD}}=45.0$ degrees. At the selected central wavelength $(\lambda=804.4 \mathrm{~nm})$ the beam enters at $\alpha_{\mathrm{CD}}=-30.42$ degrees and is diffracted at $\beta_{\mathrm{CD}}=14.58$ degrees.

- a camera introducing lateral chromatism and field distortion. In the extended model these are represented by bivariate polynomials, values at grid points being obtained from the Code $\mathrm{V}$ analysis.

A detailed presentation of the steps and equations involved in the UVES model is given in Appendix A.

\subsubsection{Comparison with ray tracing}

Without the polynomial correction for aberrations the analytical model reproduces the positional information of the Code $\mathrm{V}$ ray tracing analysis with an rms error of 8 pixels of the detector, i.e. an error of less than $0.5 \%$. Locally the genuine analytical model is much more accurate as regards curvatures, tilts and distances (see Sect. 3.2.2).

Including a polynomial correction for the aberrations and field distortions, the comparison with ray tracing shows a precision of the analytical model of better than 1 pixel anywhere in the field. Regarding line tilt, the results are in full agreement with Code V.

Since the UVES mode considered has all vital elements of a genuine echelle spectrograph and in addition the pronounced line curvature from off-plane configurations we conclude that the formalism developed in the previous Section is indeed capable of modeling the geometric aspects with the accuracy necessary for applications in the domain of calibration and data analysis.

\subsubsection{Simulated spectral formats}

One important aspect of our simulation is to show in detail the curvature of the slit images. We recall that this is made possible only by the rigorous off-plane formulation. The simulation of orders 63,76 and 98 is shown in Fig. 2.

It is interesting to note that one of the first practical applications of this analytical model was to determine the optimal slit rotation angle minimizing the slit curvature. Further simulation showed that the curvature can be minimized by rotating the slit by an angle of 5.95 degrees. In this case, for a pixel size of $15 \mu \mathrm{m}$, the slit image extends across 200 pixels while the curvature reaches a maximum value of only 1 pixel at the extremes. 


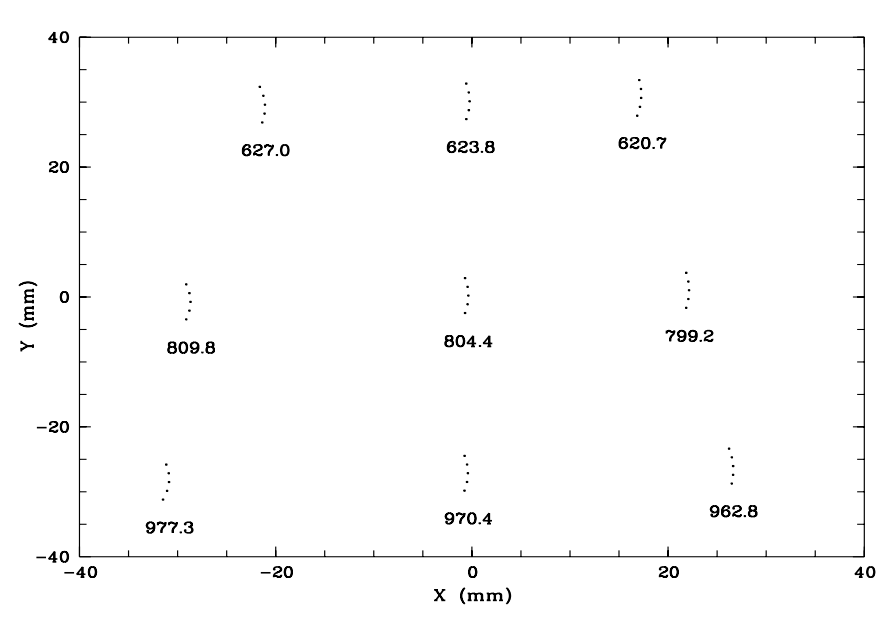

Fig. 2. Slit curvature in the red arm. The curvature is shown on a scale exaggerated by a factor 10 along the $x$-axis and 2 along the $y$-axis. Positions on the detector are in $\mathrm{mm}$ for lines taken at the limits of the free spectral range in 3 different orders (order 98 is at the top of the graph, 76 in the middle, and 63 at the bottom)

\section{Confronting the model with observational data}

\subsection{The CASPEC model}

In the following the first principle description is tested against actual arc lamp exposures from an existing spectrograph, here CASPEC in service at ESO, La Silla since 1984. With the exception of the camera, this spectrograph employs only reflective surfaces, and the distortions of this camera are very small. As an initial guess for the parameters of the model one uses engineering parameters, as they were measured at the time of instrument commissioning in January 1984, namely:

- an echelle reflection grating (31.6 grooves $/ \mathrm{mm})$. The beam enters at $\alpha_{\mathrm{E}}=-71.5$ degrees and $\gamma_{\mathrm{E}}=0$ degrees, and $\beta_{\mathrm{E}}+\alpha_{\mathrm{E}}=-13.0 \pm 0.1$ degrees, such that the output axis is oriented at $\beta_{\mathrm{E}}=58.3$ degrees, $\gamma_{\mathrm{E}}^{\prime}=0$ degrees.

- a reflective cross-disperser with 300 grooves $/ \mathrm{mm}$. The angle of incidence depends on the central wavelength, and the angle separation between incident and diffracted rays is $\beta_{\mathrm{CD}}-\alpha_{\mathrm{CD}}=45.3 \pm 0.2$ degrees.

- a camera with focal length $F_{\mathrm{CAM}}=0.288 \pm 0.003 \mathrm{~m}$. The focal length depends slightly on wavelength. Field distortions are below 1 pixel wrt a grid.

\subsubsection{Adjusting the CASPEC model}

Arc lamp calibration exposures from 3 different epochs were compared with the model, each corresponding to different configurations (detectors, central wavelength and cross-dispersers). Because the limiting factor for the accuracy of our model will be the camera distortions, and since no Code V simulations are available to compare with, we assume a threshold accuracy of 1.5 pixel rms for the model.

For each well detected calibration line the $x, y$ positions are predicted by the model using its catalog wavelength and the order number. The rms residuals between the measured and predicted positions serve to estimate the goodness of the fit. One starts with the above initial values for the optical configuration. An initial guess for $\tau_{\mathrm{DET}}$ can be obtained by geometrical measurement on the frame. An initial guess for $\alpha_{\mathrm{CD}}$ can be derived from the central wavelength by solving the dispersion equation of the cross-disperser. The following parameters are been kept at their nominal values: $\beta_{\mathrm{CD}}-\alpha_{\mathrm{CD}}=45.3$ degrees; $\beta_{\mathrm{E}}+\alpha_{\mathrm{E}}=-13.0$ degrees; the grating constants; and the detector pixel size.

The model is iteratively refined by modifying the four parameters which are found to have the largest influence:

$-\alpha_{\mathrm{CD}}$, the angle of incidence on the cross disperser,

$-\alpha_{\mathrm{E}}$ the angle of incidence on the echelle grating,

$-F_{\mathrm{CAM}}$ the focal length of the camera, and

$-\tau_{\text {DET }}$ the rotation angle of the detector.

Near the optimal solution for each configuration, respectively epoch, the model matches the observational frames to within $0.4 \pm 0.3$ pixel rms (Fig. 3). The neighborhood within which the condition of 1.5 pixel rms is fulfilled yields an uncertainty estimate for these four parameters. Table 1 presents the results. It is important to note, that the values estimated by this method on the exposures from different epochs and configurations are stable and consistent with the measured values. The value of $F_{\mathrm{CAM}}$ is not a measurement of the focal length of the camera itself, but represents the distance between the camera lens and the detector plane instead. We note that the incidence angle on the echelle grating remained stable from 1984 to 1991. The nominal value is different for epoch 1994, and although this change is within the quoted error margin, it can actually be traced to a reassembly of the instrument in 1992. This is an indication, that the predictive power of our model might be better than the conservative error estimates based on an arbitrary value of 1.5 pixel.

Certainly, this technique for controlling an instrument configuration by comparison with a model needs further investigation. However, the example shows that an accurate determination of the instrument configuration can be obtained, provided that the model includes all the important optical effects. Thus the approach through analytical models is useful to monitor the instrument stability and to predict calibration solutions, if configuration control is imposed.

\section{Wavelength calibration of echelle spectra}

In the following we take a closer look onto the practical use in wavelength calibration of the formalism developed 


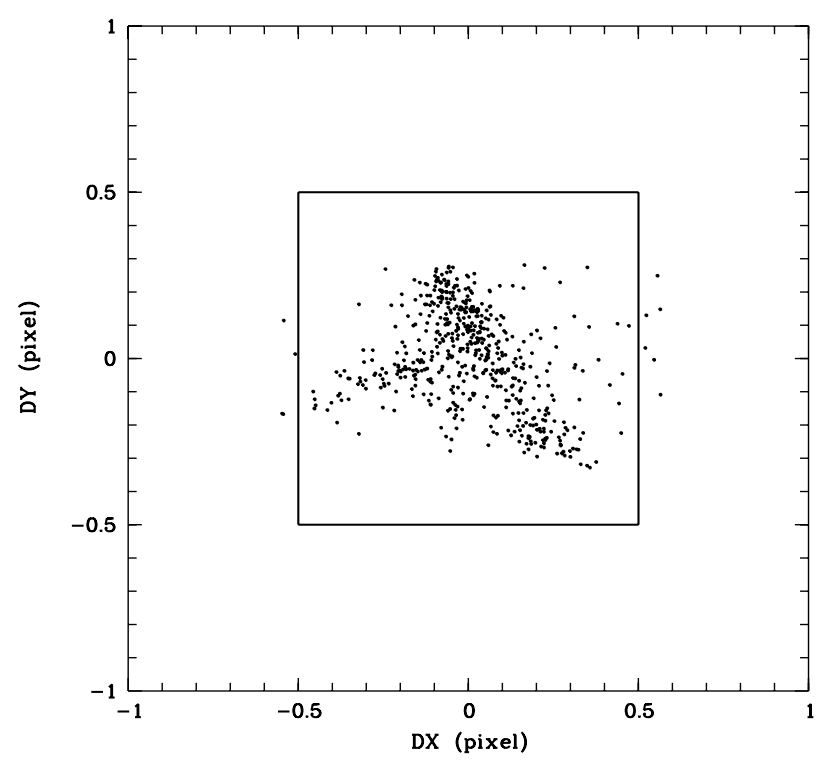

Fig. 3. Residuals between the measured positions of about 560 Th-Ar lines in the CASPEC observation $\mathrm{C}$ and the prediction from the model. The square indicates one pixel on the detector. Without a detailed analysis of the line centering methods it is not possible to relate the asymmetry of the distribution to systematics in either the model or the measurements. In any case the asymmetry is small in comparison to detector pixel size, i.e. $1 / 2$ resolution element

Table 1. Optical configuration of CASPEC as determined from a set of observations using the analytical model

\begin{tabular}{|c|c|c|c|}
\hline \begin{tabular}{l|} 
Observation \\
Date \\
Central Wav. \\
Detector \\
Cross Disperser
\end{tabular} & \begin{tabular}{|c|}
$\mathrm{A}$ \\
Jul. 1984 \\
$610 \mathrm{~nm}$ \\
$\mathrm{CCD} \# 3$ \\
300. gr. $/ \mathrm{mm}$
\end{tabular} & \begin{tabular}{|c|} 
B \\
Jan. 1991 \\
$490 \mathrm{~nm}$ \\
CCD\#16 \\
300. gr. $/ \mathrm{mm}$
\end{tabular} & $\begin{array}{c}\mathrm{C} \\
\text { Apr. } 1994 \\
600 \mathrm{~nm} \\
\text { CCD\#32 } \\
\text { 158. gr. } / \mathrm{mm}\end{array}$ \\
\hline $\begin{array}{l}\alpha_{\mathrm{CD}}(\text { deg. }) \\
\alpha_{\mathrm{E}}(\text { deg.) } \\
F_{\mathrm{CAM}}(\mathrm{m}) \\
\tau_{\text {DET }}(\text { deg. })\end{array}$ & $\begin{array}{c}28.2 \pm 2 \\
-71.2 \pm 0.7 \\
0.286 \pm 0.003 \\
-0.1 \pm 0.6\end{array}$ & $\begin{array}{c}27.0 \pm 2 \\
-71.2 \pm 0.6 \\
0.283 \pm 0.003 \\
1.85 \pm 0.35\end{array}$ & $\begin{array}{c}26.0 \pm 2.5 \\
-71.6 \pm 0.6 \\
0.292 \pm 0.003 \\
1.50 \pm 0.4\end{array}$ \\
\hline
\end{tabular}

so far and validated against two specific spectrographs. It is important to recall that we have started from text book physical principles, derived analytical equations and their simplifications for practical purposes with the primary goal to obtain robust procedures for improved calibration and data analysis.

\subsection{Generating initial solutions}

The model represents adequately all effects for off-plane spectrographs including line curvature with an accuracy close to one pixel. Because it is mostly based on linear algebra (see Appendix A), it is straight forward to produce high performance code. Such code can be used to generate accurate initial solutions of the dispersion relation using the grating positions as provided by encoders. This allows to implement fully automatic wavelength calibration procedures.

In cases where no grating positions are available, or where the zero points of such readings are unstable, one has to resort to interactive line identifications. Clearly, for these cases one will want to derive simple procedures and code. An important question therefore is how to make use of the special properties of the echelle relation in order to minimize the amount of such line identifications required to bootstrap a semi-automatic wavelength calibration procedure. In order to remain of any practical use, the simplified formalism still has to provide a local accuracy of a few resolution elements.

\subsection{Simplified forms of the echelle relation}

Using the set of equations presented in Appendix A, here with the approximation $\tan (u) \approx u$, and defining the intermediate quantities $\chi_{1}$ and $\chi_{2}$ as:

$$
\begin{aligned}
& \chi_{1}=m \lambda \sigma_{\mathrm{E}}-\sin \left(\alpha_{\mathrm{E}}\right) \\
& \chi_{2}=\cos \left(\mu_{1}\right) \chi_{1}+\sin \left(\mu_{1}\right) \sqrt{1-\chi_{1}^{2}}
\end{aligned}
$$

one derives for an in-plane spectrograph $\left(s=0, \gamma_{\mathrm{E}}=\right.$ $\left.\gamma_{\mathrm{E}}^{\prime}=0\right)$ :

$x_{\mathrm{d}}=\chi_{2} F_{\mathrm{CAM}}+x_{\mathrm{d} 0}$.

Because of the approximation $\tan (u) \approx u$ this form for $x_{\mathrm{d}}=f(\lambda)$ does not depend on the characteristics of the cross-disperser. In the absence of detector rotation (see also Hall et al. 1994) the product $m \lambda$ is constant for a given $x_{\mathrm{d}}$, i.e. a fixed column on the detector.

One can solve Eq. (26) for $\lambda$ as

$m \lambda=a_{0}+a_{1} x_{\mathrm{d}} \pm \sqrt{a_{2}+a_{3} x_{\mathrm{d}}+a_{4} x_{\mathrm{d}}^{2}}$

with the coefficients $a_{i}, i=0, . ., 4$ involving the engineering parameters. If in addition the order curvature is negligible one can simplify further to a first order polynomial.

Usually in real instruments the alignment of the detector with the dispersion direction is not perfect. A misalignment by only 1 degree on a $1 \mathrm{~K}$ pixel detector amounts to an error of about 10 pixels at the edges. Hence, it is necessary to correct either the positions or the linear echelle relation for detector rotation.

$m \lambda=a_{0}+a_{1}\left(x_{\mathrm{r}}+y_{\mathrm{r}} \tan (\alpha)\right)$.

In this case only three parameters will be needed to describe the initial relation. The value of this rotation angle 
may be determined geometrically, for example by comparing the difference in $y$ position for a given wavelength in two adjacent orders.

This naive approximation would state that y-positions depend only on the characteristics of the cross-disperser. However, it can readily be seen that even under the assumptions from the start of this section, this is an oversimplification, because the expression of the position $y_{\mathrm{d}}$ as it can be derived from the formalism in Appendix A involves both, $m \sigma_{\mathrm{E}} \lambda$ and $\sigma_{\mathrm{CD}} \lambda$ respectively. It is obvious that the $y$ positions have to depend also on the dispersion relation of the echelle grating as well, because the combination of the two gratings produces the well known order curvature in echellograms. Therefore one cannot expect a sufficiently accurate determination of the rotation angle by simply comparing $y$-positions for fixed wavelengths. If the order curvature is significant one will need more identifications, and a least-square minimization of equation 28 as presented in Appendix B. Still this model based bootstrapping of the wavelength calibration requires less initial idenfications than 2d-polynomial methods as presented by e.g. Goodrich \& Veilleux (1988) or Verschueren et al. (1997).

\section{Discussion}

In the foregoing analysis we have shown that algorithmically rather simple, yet very precise models can be constructed for spectrographs, even if significant off-plane designs are involved. The approach from first principles and its inherent predictive power allows one to quantitatively assess the degree of simplification permitted for various applications, which are at the focus of this discussion.

The most direct application of instrument models is certainly in the area of data calibration. Historically, the data calibration process has been tackled as "a cleaning from instrumental signatures". Usually one employs empirical approximations obtained from the observation of "standards" (cf. Rosa 1995). A typical example is the empirical determination of a dispersion relation by fitting a low order polynomial to a list of positions of calibration lines. Dahlem \& Rosa (1997) have shown for low dispersion, first order grating spectra from the FOS on-board HST that the dispersion relation analysis in the presence of noise, small line lists, centering errors and line blending is greatly improved by the use of optical relations. This is entirely due to the predictive power for the local curvature of a first principle derived relation, thereby avoiding the overshoot of polynomials at the boundaries of the data range.

As was shown in Sect. 5, the first principle based analytical model of echelle spectrographs can ease very significantly the tedious wavelength calibration task of 2Dechellograms. We stress the fact that it is important to perform the proper simplification of the problem in order to preserve the accuracy of the approach while limiting the complexity of the formulae to be implemented. In particular, it is compulsory to use $3 \mathrm{D}$ geometrical transformations in order to reproduce on the sub-pixel scale the observed dispersion relations of off-plane echelle designs and in the presence of detector rotation.

One can on this basis set up template based calibration procedures by providing sets of parameters with associated uncertainty ranges in order to fit the expression to actual calibration exposures pertaining to a given scientific observation. We emphasize the fact that these parameters are the engineering values such as focal length, grating constants, construction and grating angles and so forth. For the case of CASPEC one has shown in Sect. 4 how these engineering parameters can be determined and verified by adjusting the proper instrument model to observational data.

It is straight forward to conceive the next stage, i.e. predictive calibration (cf. Rosa 1995), by using contemporary values for the engineering parameters in order to provide best guess dispersion relations, which may be adjusted by small linear offsets using inexpensive control observations. Seemingly similar procedures are indeed implemented in e.g. the HST pipeline calibration. However, it is important to emphasize that in the latter case empirical calibration relations are used, which have only limited predictive power as regards the effect of varying individual engineering parameters. For example, an ambient temperature effect on the dispersion relation does not reflect itself directly in a single parameter of the 3rd order polynomial fit to this relation.

One of the main objections to the "first principle" approach has been of a practical kind, namely the lack of control of the setup of ground-based astronomical instruments. However, more rigid configuration control is now being implemented in an increasing number of observatories. It is clear, that data analysis methods based on physical models of such controlled configurations will enjoy a large gain over purely empirical methods. Furthermore, a physical model based approach removes the non-uniqueness of the relation between engineering parameters and coefficients in empirical relations. This offers operational advantages such as methods for automatic configuration control and instrument check-out.

The observational process as a whole can therefore benefit from a first principle instrument model approach during all its phases. At first, the proposer can prepare observations using model based exposure time estimators and data simulators. This is helpful for the selection of instrumental modes and exposure times suited for optimal information return. Second, the observatory controls the instrumental configuration, tests data analysis procedures, and provides calibration solutions with the help of instrument models. Thirdly, the interpretation of the data can be supported by the simulation of raw observational data for a range of target properties. 


\section{Summary}

We have developed in this article the generic optical model of two-dimensional cross-dispersed spectrographs starting from first principles. On this bases a model was formulated for the VLT instrument UVES, and its predictions have been compared with a ray tracing analysis. In this way it could be verified that local predictions of the model are in full agreement with Code V, and that the accuracy of the global geometry is only limited by the fact that we did not attempt to include the effect of aberrations and distortions. Similarly a model for the ESO La Silla instrument CASPEC was confronted with real observations in order to demonstrate the applicability of such models in two important areas, namely wavelength calibration and instrument configuration control. It has also been shown how to derive simplified, but still accurate formulations for practical use. Finally, the advantages of placing such models into operational scenarios for observatories have been discussed.

Acknowledgements. It is a pleasure to thank H. Dekker, S. D'Odorico, J.L. Lizon, and S. Randich for helpful hints and fruitful discussions. We are particularly grateful to B. Delabre for providing us with the Code- $\mathrm{V}$ analysis and engineering data.

\section{A. Model for the CASPEC and UVES spectrographs}

This appendix describes the model used for the CASPEC and UVES spectrographs. The optical design of the two instruments is similar enough to justify the use of the same description for both instruments. The analytical model taking into account the 3-dimensional grating equations involves the following successive steps:

- Slit rotation by an angle $\tau_{0}$ and collimation by $F_{\mathrm{COL}}$

- Diffraction by the echelle grating

- Rotation by $-\mu_{1}$ about $Y$

- Rotation by $-\nu_{1}$ about $X$

- Rotation by -90 degrees about $Z$

- Diffraction by the cross-disperser

- Rotation by 90 degrees about $Z$

- Rotation by $\nu_{1}$ about $X$

- (We assume $\mu_{1}=-\mu_{2}$, and skip two rotations about $Y)$

- Rotation by $-\nu_{2}$ about $X$

- Projection onto the detector plane

- Offset in position $\left(x_{\mathrm{d} 0}, y_{\mathrm{d} 0}\right)$

- Rotation of the detector by an angle $\tau_{2}$.

The $x$-axis of our coordinate system is perpendicular to the grooves of the echelle grating, the $z$-axis normal to the surface. Nomenclature is as in Sect. 2 of the main article. In addition, $s$ is the position on the slit, $F_{\mathrm{COL}}$ the focal length of the collimator, $\tau_{0}$ the angle of rotation of the slit.
For practical purposes we construct a 4-dim vector using the wavelength $\lambda$ and the projections $x, y, z$. The initial ray has the directions and projections

$$
\begin{aligned}
\gamma & =\gamma_{\mathrm{E}}+\cos \left(\tau_{0}\right) \arctan \left(s / F_{\mathrm{COL}}\right) \\
\alpha & =\alpha_{\mathrm{E}}+\sin \left(\tau_{0}\right) \arctan \left(s / F_{\mathrm{COL}}\right) \\
(x, y, z) & =(\sin (\alpha) \cos (\gamma), \sin (\gamma), \cos (\alpha) \cos (\gamma))
\end{aligned}
$$

The list of matrices for the above sequence of operations is given below, where after each pass through a grating the $z$-component of the vector is determined from the normalization relation

$z^{2}=1-x^{2}-y^{2}$

$M_{\mathrm{E}}=\left(\begin{array}{cccc}1 & 0 & 0 & 0 \\ \frac{m}{\sigma_{\mathrm{E}}} & -1 & 0 & 0 \\ 0 & 0 & 1 & 0 \\ 0 & 0 & 0 & 1\end{array}\right)$

$R_{-\mu_{1} / y}=\left(\begin{array}{cccc}1 & 0 & 0 & 0 \\ 0 & \cos \mu_{1} & 0 & -\sin \mu_{1} \\ 0 & 0 & 1 & 0 \\ 0 & \sin \mu_{1} & 0 & \cos \mu_{1}\end{array}\right)$

$R_{-\nu_{1} / x}=\left(\begin{array}{cccc}1 & 0 & 0 & 0 \\ 0 & 1 & 0 & 0 \\ 0 & 0 & \cos \nu_{1} & -\sin \nu_{1} \\ 0 & 0 & \sin \nu_{1} & \cos \nu_{1}\end{array}\right)$

$R_{-\frac{\pi}{2} / z}=\left(\begin{array}{cccc}1 & 0 & 0 & 0 \\ 0 & 0 & -1 & 0 \\ 0 & 1 & 0 & 0 \\ 0 & 0 & 0 & 1\end{array}\right)$

$M_{\mathrm{CD}}=\left(\begin{array}{cccc}1 & 0 & 0 & 0 \\ \frac{1}{\sigma_{\mathrm{CD}}} & -1 & 0 & 0 \\ 0 & 0 & 1 & 0 \\ 0 & 0 & 0 & 1\end{array}\right)$

$R_{\frac{\pi}{2} / z}=\left(\begin{array}{cccc}1 & 0 & 0 & 0 \\ 0 & 0 & 1 & 0 \\ 0 & -1 & 0 & 0 \\ 0 & 0 & 0 & 1\end{array}\right)$

$R_{\nu_{1} / x}=\left(\begin{array}{cccc}1 & 0 & 0 & 0 \\ 0 & 1 & 0 & 0 \\ 0 & 0 & \cos \nu_{1} & \sin \nu_{1} \\ 0 & 0 & -\sin \nu_{1} & \cos \nu_{1}\end{array}\right)$

$R_{-\nu_{2} / x}=\left(\begin{array}{cccc}1 & 0 & 0 & 0 \\ 0 & 1 & 0 & 0 \\ 0 & 0 & \cos \nu_{2} & -\sin \nu_{2} \\ 0 & 0 & \sin \nu_{2} & \cos \nu_{2}\end{array}\right)$.

One can at this stage either determine the complete expression of $x_{\mathrm{d}}$ and $y_{\mathrm{d}}$ 


$$
\begin{aligned}
& x_{\mathrm{d}}=F_{\mathrm{CAM}} \frac{x}{z}+x_{\mathrm{d} 0} \\
& y_{\mathrm{d}}=F_{\mathrm{CAM}} \frac{y}{\sqrt{x^{2}+z^{2}}}+y_{\mathrm{d} 0}
\end{aligned}
$$

or make the approximation $\tan (u) \approx u$ and obtain

$$
\begin{aligned}
& x_{\mathrm{d}} \approx F_{\mathrm{CAM}} x+x_{\mathrm{d} 0} \\
& y_{\mathrm{d}} \approx F_{\mathrm{CAM}} y+y_{\mathrm{d} 0} .
\end{aligned}
$$

Finally one applies the rotation of the detector:

$$
\begin{aligned}
& x_{\mathrm{r}}=x_{\mathrm{d}} \cos \left(\tau_{2}\right)-y_{\mathrm{d}} \sin \left(\tau_{2}\right) \\
& y_{\mathrm{r}}=x_{\mathrm{d}} \sin \left(\tau_{2}\right)+y_{\mathrm{d}} \cos \left(\tau_{2}\right) .
\end{aligned}
$$

\section{B. Evaluation of the rotated echelle relation}

In order to obtain an analytical least-squares solution we use the simplified rotated echelle relation. The residual (measurement - model) of a given line of index $i \quad(i=$ $1 \ldots N)$ at position $\left(x_{i}, y_{i}\right)$ and of wavelength $\lambda_{i}$, and the relative order number $p_{i}$, is given by

$$
R_{i}=\left(m+p_{i}\right) \lambda_{i}-a-b\left(x_{i}+y_{i} \tan \alpha\right) .
$$

One has to consider the case where the order number $m$ is also an unknown quantity since it has useful practical applications. We rewrite, using $\tan (\alpha)=c / b$

$$
R_{i}=\left(m+p_{i}\right) \lambda_{i}-a-b x_{i}-c y_{i}
$$

By determining the partial derivatives of $R_{i}^{2}$ with respect to each parameter $(m, a, b, c)$ we obtain a system of the form $A x=B$ with $A$ a $4 \times 4$ matrix and $B$ a vector such as

$$
A=\left(\begin{array}{cccc}
S_{\lambda^{2}} & -S_{\lambda} & -S_{x \lambda} & -S_{y \lambda} \\
-S_{\lambda} & 1 & S_{x} & S_{y} \\
-S_{x \lambda} & S_{x} & S_{x^{2}} & S_{x y} \\
S_{y \lambda} & S_{y} & S_{x y} & S_{y^{2}}
\end{array}\right)
$$

$B=\left[-S_{p \lambda^{2}}, S_{p \lambda}, S_{x p \lambda}, S_{y p \lambda}\right]$

with the quantities $S_{x}$, etc... being defined as

$$
S_{x}=\sum_{i=1}^{N} x_{i}
$$

$$
\begin{aligned}
& S_{x y}=\sum_{i=1}^{N} x_{i} y_{i} \\
& S_{x^{2}}=\sum_{i=1}^{N} x_{i}^{2}
\end{aligned}
$$

and similar formulae for $S_{y}, S_{\lambda}, S_{p \lambda}, S_{y \lambda}, S_{x p \lambda}, S_{y p \lambda}, S_{y^{2}}$, $S_{\lambda^{2}}, S_{p^{2} \lambda^{2}}, S_{p \lambda^{2}}$.

The full system was solved using mathematical packages, and solutions for subsets with fixed parameters were determined with a view towards robust techniques for practical calibration procedures.

\section{References}

Ballester P., 1994, A\&A 206, 1108

Ballester P., 1996, in: Proc. High Resolution Data Processing Workshop, Iye M., Takata T. \& Wampler E.J. (eds.), Subaru Telescope Technical Report No. 55, p. 17

Bushouse H., Rosa M.R., Mueller Th., 1995, in: Astronomical Data Analysis Software and Systems, Shaw R.A., Payne H.E. \& Hayes J.J.E. (eds.), ASP Conf. Ser. 77, p. 345

Dahlem M., Rosa M.R., 1997, HST FOS Instrument Science Report Ser., STScI (in press)

D'Odorico S., 1997, Proceedings of the ESO Workshop "The Early Universe with the VLT", Bergeron J. (ed.). Springer Verlag (in press)

Goodrich R.W., Veilleux S., 1988, PASP 100, 1572

Hall J.C., Fulton E.E., Huenemoerder D.P., Welty A.D., Neff J.E., 1994, PASP 106, 315

Hasan H., Burrows C.J., 1994, in: Calibrating Hubble Space Telescope, Blades J.C. \& Osmer S.J. (eds.), STScI, p. 395

Hensberge H., Verschueren W., 1990, A\&A 240, 216

Randich S., Pasquini L., 1997, ESO CASPEC Operating Manual

Rosa M.R., 1994, in: Calibrating Hubble Space Telescope, Blades J.C. \& Osmer S.J. (eds.), STScI, p. 190

Rosa M.R., 1995, in: Calibrating and Understanding HST and ESO Instruments, Benvenuti P. (ed.), ESO, p. 43

Schroeder 1987, "Astronomical Optics", AP, San Diego

Verschueren W., Brown A.G.A., Hensberge H., David M., Lepoole R.S., de Geus E.J., de Zeeuw P.T., 1997, PASP (in press) 